\title{
Regeneration of Peach [Prunus persica (L.) Batsch] Rootstock Cultivars from Cotyledons of Mature Stored Seed
}

\author{
M.R. Pooler and R. Scorza \\ U.S. Department of Agriculture, Agricultural Research Service, Appalachian \\ Fruit Research Station, 45 Wiltshire Road, Kearneysville, WV 25430
}

Additional index words. benzyladenine, indole-3-butyric acid, organogenesis, thidiazuron, tissue culture

\begin{abstract}
Shoots were regenerated from cotyledons of mature stored seed of three peach rootstock cultivars ('Flordaguard', 'Nemared', and 'Medaguard'). Shoot regeneration rates were highest when cotyledons were cultured for 3 weeks in darkness on Murashige and Skoog (MS) medium with $2.5 \%$ sucrose and a combination of IBA $(1.25$ or $2.5 \mu \mathrm{M})$ and TDZ (6.25 or 12.5 $\mu \mathrm{M})$. Regeneration rates for 'Flordaguard', 'Nemared', and 'Nemaguard' were as high as $60 \%, 33 \%$, and $6 \%$, respectively. Length of seed storage ( 1 to 3 years) did not affect regeneration rates. Seventy percent of regenerated shoots produced rooted plants. This regeneration method is rapid and simple, and stored seed can be used yearround. It may be a useful regeneration system for gene transfer in seed-propagated peach rootstocks. Chemical names used: 5 indole-3-butyric acid (IBA); thidiazuron (TDZ).
\end{abstract}

One requirement for a successful transformation system is reliable high-frequency regeneration. Previous peach regeneration methods have depended on time-consuming, multistepped embryo production (Hammerschlag et al., 1985; Raj Bhansali et al., 1990) or have required stage-specific immature cotyledons (Mante et al., 1989). Our study reports shoot regeneration from cotyledons of stored seed of mature fruit from the commercially important peach rootstocks 'Flordaguard', 'Nemaguard', and 'Nemared'.

\section{Materials and Methods}

Mature 'Nemared' peach seeds were obtained from a 1991 crop grown in Kearneysville, W.Va., or from 1992 and 1993 crops from Foundation Plant Materials Service (FPMS), Univ. of California, Davis 'Nemaguard' seeds were obtained from 1991 and 1992 crops grown in Kearneysville, or from a 1993 crop from FPMS. 'Flordaguard' seeds from 1991 and 1992 crops were supplied by W.B. Sherman, Univ. of Florida, Gainesville. Seeds in their endocarps were stored in paper or mesh bags at $4 \mathrm{C}$ until use in 1993.

Basal culture medium was MS salts and vitamins (Murashige and Skoog, 1962) with a $\mathrm{pH}$ of 6.0 and $2.5 \%$ sucrose. Initial experiments using 1992 'Flordaguard' seeds in a

Received for publication 7 Mar. 1994. Accepted for publication 25 Nov. 1994. Mention of a trademark, proprietary product, or vendor does not constitute a guarantee or warranty of the product by the U.S. Dept. of Agriculture and does not imply its approval to the exclusion of other products or vendors that also may be suitable. We thank J.M. Cordts for his technical assistance. The cost of publishing this paper was defrayed in part by the payment of page charges. Under postal regulations, this paper therefore must be hereby marked advertisement solely to indicate this fact. factorial of (in $\mu \mathrm{M}$ ) $0,1.25,2.5$, or $5 \mathrm{IBA}$ and $3.12,6.25,12.5$, or $25 \mathrm{TDZ}$ gave the highest regeneration rates with $1.25 \mathrm{IBA}+6.25 \mathrm{TDZ}$ (34\%), 2.5 IBA + 12.5 TDZ (53\%), and 5.0 $\mathrm{IBA}+6.25 \mathrm{TDZ}(38 \%)$. Based on these results, regeneration medium was prepared by supplementing basal medium with a combination of 1.25 or $2.5 \mu \mathrm{M}$ IBA and 6.25 or $12.5 \mu \mathrm{M}$ TDZ.

Maintenance medium was basal medium supplemented with $0.1 \mu \mathrm{M}$ IBA and $1.0 \mu \mathrm{M}$ benzyladenine (BA). Rooting medium was half-strength basal medium supplemented with $0.7 \% \mathrm{TC}$ agar and poured into $100 \times 20-\mathrm{mm}$ petri plates $(25 \mathrm{ml}$ per plate).

Seeds were removed from their endocarps immediately before use. The seeds were disinfested $1 \mathrm{~min}$ in 70\% ethanol, $15 \mathrm{~min}$ in $0.75 \%$ sodium hypochlorite plus $0.01 \%$ Tween-20 (Sigma Chemical Co., St. Louis), and rinsed three times with sterile distilled water. Seeds then were soaked overnight in sterile distilled water. The seedcoats, embryonic axes, and tissue immediately surrounding the embryonic axis were removed with a scalpel and discarded. Cotyledons were oriented with abaxial surfaces in contact with the regeneration medium (eight cotyledons per plate).

\section{Results and Discussion}

In contrast to results with immature peach seeds (Mante et al., 1989), preliminary experiments with mature seed indicated that 3 weeks of darkness was necessary for regeneration. Therefore, explants were cultured at $24 \mathrm{C}$ in darkness for 3 weeks, then moved to a 16 -h photoperiod under 50 to $55 \mu \mathrm{mol} \cdot \mathrm{m}^{-2} \cdot \mathrm{s}^{-1}$ of mixed warm-white fluorescent and Vita-light full-spectrum fluorescent lamps (Duro-Test Corp., North Bergen, N.J.). After 1 to 2 weeks under light, when regenerating shoots were $5 \mu_{\mathrm{M}}$ IBA. All media were solidified with $\approx 0.5 \mathrm{~cm}$ long, the shoots were excised from the cotyledon and transferred to maintenance medium. When shoots were $3 \mathrm{~cm}$ tall, they were rooted by dipping the freshly cut base of the shoot in $2.5 \mathrm{~mm}$ IBA and transferring it to rooting medium. About $80 \%$ of the shoots rooted within 3 weeks, and plantlets were transferred to Magenta boxes (Sigma Chemical Co.) containing sterile vermiculite supplemented with half-strength basal medium. Few additional plants rooted after 3 weeks. Lids were gradually (over 1 week) removed from the Magenta boxes, and plants were planted in Promix BX potting medium (Premier Brands, Yonkers, N.Y.), sprayed with AntiStress 2000 (Polymer Ag, Fresno, Calif.), and transferred to a greenhouse.

Data were analyzed using the general linear model procedure (SAS Institute, 1989). Mean regeneration rates were calculated for each of the growth-regulator concentration combinations (GRCC) over all eight seed sources combined, for each seed source in each specific GRCC over all GRCC combined, for each GRCC within each cultivar, for seed age over all GRCC and cultivars, and for each cultivar over all GRCC and seed age.

Regenerating cotyledons are characterized by a swelling of the cotyledon at the proximal end after 1 to 2 weeks in culture, followed in some cases by callus production and shoot development from the proximal end. Cotyledons with little callus development generally produced only one to two large shoots per cotyledon, whereas cotyledons with a large callus mass produced $\leq 20$ small shoots. Regenerating cotyledons yielded one to five transferrable shoots per cotyledon, with a shoot-toestablished-plant rate of $\approx 70 \%$.

There were significant differences in regeneration rates of cotyledons from all eight seed lots (Table 1). However, when data from all genotypes were pooled within each year and these pooled data were compared between the 3 years, there were no significant differences in regeneration rates, indicating that within this 3-year study, seed age did not affect regeneration. The effect of the four GRCC was not significant, either overall or within cultivars or individual seed lots.

The most striking difference in regeneration was between the seed source cultivars. The average regeneration rate for 'Flordaguard'

Table 1. Average percentage of peach cotyledons forming shoots for eight seed sources over four IBA-TDZ concentration combinations.

\begin{tabular}{llllr}
\hline \multicolumn{2}{c}{ Seed source } & & \multicolumn{2}{c}{ Avg regeneration $(\%)$} \\
\cline { 5 - 5 } Cultivar & Year & & Year & Overall \\
\hline Flordaguard & 1991 & & $59.9 \mathrm{a}^{\mathrm{z}}$ & $50.5 \mathrm{a}^{\mathrm{z}}$ \\
& 1992 & & $42.7 \mathrm{~b}$ & \\
Nemaguard & 1991 & & $6.1 \mathrm{c}$ & \\
& 1992 & & $3.8 \mathrm{c}$ & $5.2 \mathrm{c}$ \\
& 1993 & & $6.3 \mathrm{c}$ & \\
Nemared & 1991 & & $4.4 \mathrm{c}$ & \\
& 1992 & & $32.6 \mathrm{~b}$ & $17.7 \mathrm{~b}$ \\
& 1993 & & $18.7 \mathrm{c}$ & \\
\hline
\end{tabular}

${ }^{2}$ Mean separation within column by Duncan's multiple range test at $P \leq 0.05$. Each treatment consisted of five plates with eight cotyledons per plate. There were no significant differences between growthregulator concentration combinations. 
over all seed lots and media combinations was $51 \%$; the rate for 'Nemared' and 'Nemaguard' reached only $18 \%$ and $5 \%$, respectively (Table 1). All three rootstocks were selected for resistance to root-knot nematodes, and all share $P$. davidiana (Carr.) Franch. as an ancestor. 'Nemared' is an $F_{3}$ seedling of 'Nemaguard' $x$ a redleaf seedling (Ramming and Tanner, 1983). 'Flordaguard' is the result of a complex series of crosses that did not include 'Nemaguard' (Sherman et al., 1991). The differences in regeneration may reflect these differences in genetic relationships.

As indicated by Mante et al. (1989), plant regeneration from cotyledons is faster and requires fewer steps than regeneration from embryo-derived callus (Hammerschlag et al., 1985). The ability to regenerate shoots from mature peach seeds stored for $\geq 3$ years allows year-round experimentation. The regeneration system that we have presented may provide an alternate method for genetic manipulation of rootstock varieties through transformation of regenerating shoots. Although transformation of seed-derived material is of limited use in improving vegetatively propagated peach scion cultivars, it could have an impact on the development of new seed-propagated rootstock varieties and the feral seed-propagated lines that are used in some countries (e.g., Perez et al., 1993).

\section{Literature Cited}

Hammerschlag, F.A., G. Bauchan, and R. Scorza. 1985. Regeneration of peach plants from callus derived from immature embryos. Theor. Appl. Genet. 70:248-251.

Mante, S., R. Scorza, and J.M. Cordts. 1989. Plant regeneration from cotyledons of Prunus persica, Prunus domestica, and Prunus cerasus. Plant Cell, Tissue Organ Cult. 19:1-11.

Murashige, T. and F. Skoog. 1962. A revised medium for rapid growth and bioassays with tobacco tissue cultures. Physiol. Plant. 15:473-497.

Perez, S., S. Montes, and C. Mejia. 1993. Analysis of peach germplasm in Mexico. J. Amer. Soc. Hort. Sci. 118:519-524.

Raj Bhansali, R., J.A. Driver, and D.J. Durzan. 1990. Rapid multiplication of adventitious somatic embryos in peach and nectarine by secondary embryogenesis. Plant Cell Rpt. 9:280284.

Ramming, D.W. and O. Tanner. 1983. 'Nemared' peach rootstock. HortScience 18:376.

SAS Institute. 1989. SAS/STAT user's guide. Version 6. 4th ed. vol. 1. SAS Institute, Cary, N.C.

Sherman, W.B., P.M. Lyrene, and R.H. Sharpe. 1991. 'Flordaguard' peach rootstock. HortScience 26:427-428. 